\title{
AFM topography and friction studies of hydrogen-bonded bilayers of functionalized alkanethiols $\dagger$
}

\author{
Joost te Riet, ${ }^{a b}$ Tim Smit, ${ }^{a}$ Michiel J. J. Coenen, ${ }^{a}$ Jan W. Gerritsen, ${ }^{a}$ Alessandra Cambi, ${ }^{b}$ \\ Johannes A. A. W. Elemans, ${ }^{* a}$ Sylvia Speller ${ }^{a}$ and Carl G. Figdor $\dagger^{b}$
}

Received 19th March 2010, Accepted 6th June 2010

DOI: 10.1039/c0sm00130a

\begin{abstract}
Atomic Force Microscope (AFM) assisted nanografting was exploited to construct-in a liquid-well-defined nanoscale patches of amino- and carboxyl-terminated alkanethiols in a SAM matrix on an atomically flat gold (Au(111)) surface. By means of AFM the nanografted patches of these thiols were studied in detail by a combination of height and quantitative lateral friction measurements. The functionalized thiols form well-ordered self-assembled bilayers, with uniformly oriented and packed molecules, consisting of a first layer adsorbed to the gold surface via thiol-gold bonds, and a second layer stably bound to the first via $\mathrm{COOH}-\mathrm{HOOC}$ or $\mathrm{NH}_{2}-\mathrm{H}_{2} \mathrm{~N}$ intermolecular hydrogen bonds.
\end{abstract}

Amino- and carboxyl-terminated self-assembled monolayers (SAMs) represent ideal platforms for chemically and biologically functionalized surfaces, mainly because of their capability to react and/or interact with other chemical functional groups via covalent coupling, or via non-covalent hydrogen bonding and electrostatic interactions. From structural studies it is known that alkanethiols, including amino- and carboxyl-terminated ones, form SAMs on gold surfaces with their hydrocarbon chains oriented in an all-trans conformation with a lattice constant of $\sim 5.0 \AA$. They are predominantly organized in a $(\sqrt{3} \times \sqrt{ } 3) R 30^{\circ}$-based structure and a $c(4 \times 2)$ superlattice in which the hydrocarbon chains are closely packed and tilted under an angle of $\sim 30^{\circ}$ with respect to the surface normal. ${ }^{1-6}$ However, many conflicting results have been reported for the characterization of highly ordered SAMs of carboxyl-terminated alkanethiols, in contrast to for example unfunctionalized thiols. In particular the proper experimental conditions for the construction of the functionalized SAMs continues to be a topic of debate. ${ }^{6-10}$ In addition, in contact angle measurements different wettabilities of SAMs of amino- and carboxyl-terminated alkanethiols have been observed, indicating the presence of hydrophilic but also hydrophobic top surfaces. ${ }^{6,7}$ Scanning tunneling microscopy (STM) and infrared spectroscopy measurements on dry carboxyl-terminated SAMs prepared from an ethanolic solution revealed in some cases a high

${ }^{a}$ Department of Scanning Probe Microscopy, Institute for Molecules and Materials, Radboud University Nijmegen, Heyendaalseweg 135, 6525 AJ Nijmegen, The Netherlands.E-mail: j.elemans@science.ru.nl; Fax: +31 24365 2190; Tel: +31 243653099

${ }^{b}$ Department of Tumor Immunology, Nijmegen Centre for Molecular Life Sciences, Radboud University Nijmegen Medical Centre, Geert Grooteplein Zuid 28, 6525 GA Nijmegen, The Netherlands

$\uparrow$ Electronic supplementary information (ESI) available: Experimental procedures and Supplementary Figures S1-S3. See DOI: $10.1039 / \mathrm{c} 0 \mathrm{sm} 00130 \mathrm{a}$

\$ These authors contributed equally. degree of disorder. The quality of such SAMs strongly improved upon the addition of acetic acid, ${ }^{8,9}$ occasionally with $10 \%$ of water, ${ }^{11}$ or with $\mathrm{CF}_{3} \mathrm{COOH}$ followed by rinsing with an aqueous $\mathrm{NH}_{4} \mathrm{OH}$ solution. ${ }^{7}$ The observed disorder in the SAMs was partially attributed to the occurrence of intermolecular hydrogen bonding between the carboxyl-termini, which could be in the form of head-to-head dimers of molecules within the layer, as dimers with single molecules on top of the monolayer, or as linear chains of neighboring COOH-groups. ${ }^{7,8}$

SAM formation of $\mathrm{NH}_{2}$ - and $\mathrm{COOH}$-terminated alkanethiols can be studied by nanografting, a method in which nanosized structures are fabricated by using an AFM cantilever to create patterns on a surface., ${ }^{4,12}$ During the nanografting procedure, patches of thiol molecules are locally 'shaved' away with an AFM tip from a SAM matrix on an ultraflat gold substrate. ${ }^{13}$ The exposed gold patches are subsequently refilled with other thiol molecules from a supernatant solution, resulting in a nanografted patch. The same cantilever is used to graft and to image, thus all procedures are carried out in situ and under liquid. During the nanografting, the thiols self-assemble in a spatially confined environment, which strongly reduces the amount of disorder present in the resulting nanografted patch. ${ }^{14}$ We have recently shown that these highly ordered patches can subsequently serve as ideal samples to study the molecular packing and orientation of alkanethiols at the molecular level in a liquid environment. ${ }^{13}$ By AFM topography imaging, the nanografted patches can be studied with quantitative atomic height resolution, with the matrix SAM and atomic gold steps serving as an internal calibration. In addition to the topography, probing the lateral friction of the nanografted patches by the AFM tip provides insight in the composition and specific chemical properties of the SAM.,12,13,15-20

In this communication we describe the characterization of layers of carboxyl- and amino-functionalized alkanethiols on gold in a 2-butanol or ethanol supernatant solution, and by combining nanografting and lateral friction measurements we will reveal the effect of intermolecular hydrogen bonding on the formation of stable monolayer and bilayer structures of these thiols.

Using the nanografting method, ${ }^{4} 500 \times 500 \mathrm{~nm}^{2}$ patches of 11-amino-1-undecanethiol ( $\left.\mathrm{HSC}_{11} \mathrm{NH}_{2}\right)$, 11-mercapto-1-undecanoic acid $\left(\mathrm{HSC}_{10} \mathrm{COOH}\right)$ and 16-mercapto-1-hexadecanoic acid $\left(\mathrm{HSC}_{15} \mathrm{COOH}\right)$ were created from a 2-butanolic solution of the thiols in a matrix $\mathrm{SAM}$ of 1 -octanethiol $\left(\mathrm{HSC}_{8}\right)$ on an atomically flat $\mathrm{Au}(111)$ surface (see Table 1 for nomenclature). Each of these experiments was performed under strictly controlled conditions, the only variable between the patches being the thiol molecule grafted. $\mathrm{HSC}_{11} \mathrm{NH}_{2}$ and $\mathrm{HSC}_{11} \mathrm{COOH}$ have a similar chain length and if they self-assemble at the expected $\sim 30^{\circ}$ tilt $^{1}$ with respect to the surface normal of the gold, the apparent height of their patches should be 
Table 1 List of molecules that were used for the formation of SAMs and nanografted patches in our experiments

\begin{tabular}{llll}
\hline Chemical name & Molecular formula & Abbreviated as & Molecular length at 30 tilt/nm $^{a}$ \\
\hline 1-Octanethiol & $\mathrm{HS}-\left(\mathrm{CH}_{2}\right)_{7}-\mathrm{CH}_{3}$ & $\mathrm{HSC}_{8}$ & 1.19 \\
11-Mercapto-1-undecanol & $\mathrm{HS}-\left(\mathrm{CH}_{2}\right)_{11}-\mathrm{OH}$ & $\mathrm{HSC}_{11} \mathrm{OH}$ & 1.61 \\
11-Amino-1-undecanethiol & $\mathrm{HS}-\left(\mathrm{CH}_{2}\right)_{11}-\mathrm{NH}_{2}$ & $\mathrm{HSC}_{11} \mathrm{NH}_{2}$ & 1.62 \\
8-Mercapto-1-octanoic acid & $\mathrm{HS}-\left(\mathrm{CH}_{2}\right)_{7}-\mathrm{COOH}$ & $\mathrm{HSC}_{7} \mathrm{COOH}$ & 1.28 \\
11-Mercapto-1-undecanoic acid & $\mathrm{HS}-\left(\mathrm{CH}_{2}\right)_{10}-\mathrm{COOH}$ & $\mathrm{HSC}_{10} \mathrm{COOH}$ & 1.58 \\
12-Mercapto-1-dodecanoic acid & $\mathrm{HS}-\left(\mathrm{CH}_{2}\right)_{11}-\mathrm{COOH}$ & $\mathrm{HSC}_{11} \mathrm{COOH}$ & 1.71 \\
15-Mercapto-1-pentadecanoic acid & $\mathrm{HS}-\left(\mathrm{CH}_{2}\right)_{14}-\mathrm{COOH}$ & $\mathrm{HSC}_{14} \mathrm{COOH}$ & 2.01 \\
16-Mercapto-1-hexadecanoic acid & $\mathrm{HS}-\left(\mathrm{CH}_{2}\right)_{15} \mathrm{COOH}$ & $\mathrm{HSC}_{15} \mathrm{COOH}$ & 2.14 \\
1-Hexadecanoic acid & $\mathrm{CH}_{3}-\left(\mathrm{CH}_{2}\right)_{14}-\mathrm{COOH}$ & $\mathrm{C}_{15} \mathrm{COOH}$ & $\mathrm{n} / \mathrm{a}$ \\
1-Octadecanoic acid & $\mathrm{CH}_{3}-\left(\mathrm{CH}_{2}\right)_{16}-\mathrm{COOH}$ & $\mathrm{C}_{17} \mathrm{COOH}$ & $\mathrm{n} / \mathrm{a}$
\end{tabular}

${ }^{a}$ The calculated lengths are those of alkanethiols bound on $\mathrm{Au}(111)$ along the surface normal, assuming a $30^{\circ}$ tilt of the carbon chain with the surface normal, and including the $\mathrm{S}-\mathrm{Au}$ binding distance of $0.23 \mathrm{~nm}$.

similar (Table 1). However, the topographies of the nanografted patches of $\mathrm{HSC}_{11} \mathrm{NH}_{2}$ and $\mathrm{HSC}_{10} \mathrm{COOH}$ are clearly much higher than that of the $\mathrm{HSC}_{8}$ matrix (Fig. 1A and $\mathrm{B}$ (top panels)), whereas earlier research has shown that the nanografted patch of the equally sized thiol $\mathrm{HSC}_{11} \mathrm{OH}$ is just slightly higher $(0.19 \pm 0.06 \mathrm{~nm}) .{ }^{13}$ Remarkably, the patches of the $\mathrm{NH}_{2}$ and $\mathrm{COOH}$-terminated alkanethiols are even 2.1 and 1.5 times higher than expected. A different orientation of these thiols on the surface cannot explain this substantially higher apparent height. Interestingly, also the lateral friction of their nanografted patches appears to be $3-4$ times higher (Fig. 1E) than that of a patch of $\mathrm{HSC}_{11} \mathrm{OH}(1.36 \pm 0.47 \mathrm{nN}){ }^{13}$
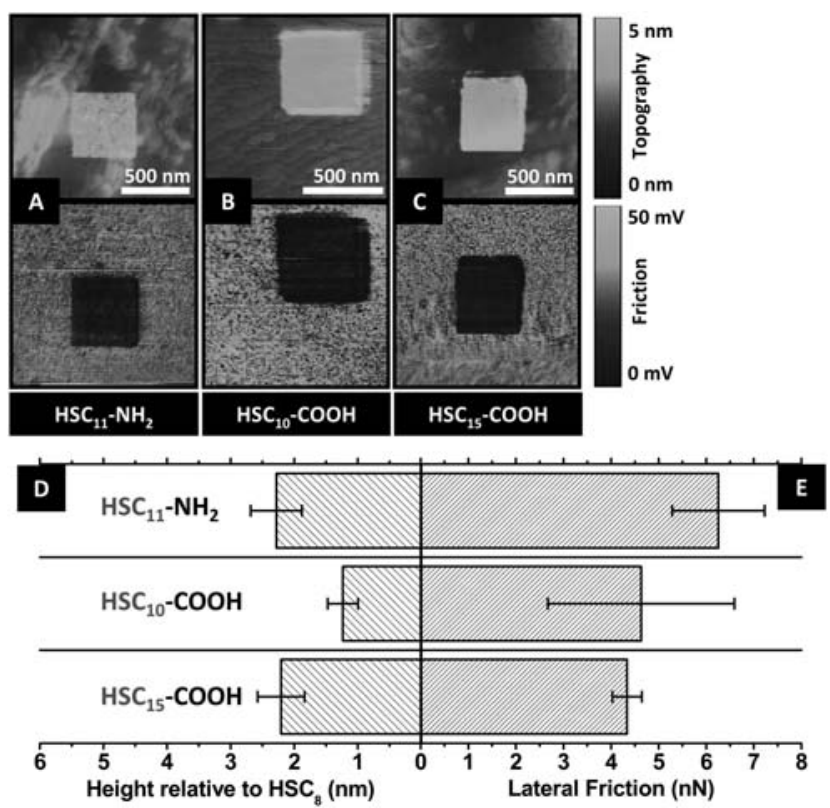

Fig. 1 AFM topography (top row) and friction images (retrace, bottom row) of nanografted patches of thiols with various endgroups in a matrix SAM of $\mathrm{HSC}_{8}$ : (A) $\mathrm{HSC}_{11} \mathrm{NH}_{2}$, (B) $\mathrm{HSC}_{10} \mathrm{COOH}$, and (C) $\mathrm{HSC}_{15} \mathrm{COOH}$. All images were recorded in a 2-butanol subphase, with a normal load force of $\sim 6 \mathrm{nN}$ and at a scan speed of $\sim 4 \mu \mathrm{m} \mathrm{s}^{-1}$. (D) Relative heights and (E) corresponding lateral friction values of the different nanografts, displayed in bar diagrams. The friction of the $\mathrm{HSC}_{8}$ matrix is $0.75 \pm 0.33 \mathrm{nN}$. Note that for the retrace friction images a darker color implies higher friction.
As explanation for these observations, we propose in the case of patches of $\mathrm{HSC}_{11} \mathrm{NH}_{2}$ and $\mathrm{HSC}_{10} \mathrm{COOH}$ the formation of bilayer structures, occurring via intermolecular hydrogen bonding between the amino or carboxylic acid headgroups of the surface-bound thiols and thiols from solution. Bilayer formation can readily explain the higher appearance of the patches in the topography, and also the observed higher friction values are in line with such a model. As a result of the relatively weak link between the two layers, which is based on non-covalent hydrogen bonding interactions, it can be expected that the top layer is substantially more dynamic than the bottom one, thereby exhibiting more packing defects and a lower stability upon exerting pressure by the scanning of the AFM tip. All these effects can contribute to a higher lateral friction. However, in the case of these bilayers the penetration of the tip in the top layer seems to be minimal at the used imaging load of $6 \mathrm{nN}$, because even a 4-fold increase in load did not significantly alter the observed height of the nanografted patches.

To investigate the proposed bilayer formation in more detail, a series of $\mathrm{HSC}_{n} \mathrm{COOH}$ alkanethiols ( $n=7,10,11,14$ and 15) was grafted. The relative heights of the nanografted patches (compared to $\mathrm{HSC}_{8}$ ) increase linearly with the length of the molecule (Fig. 2A). In addition, the mean relative heights match a model in which the first layer has a $30^{\circ}$ tilt, and the second layer an extra $\sim 30^{\circ}$ tilt compared to the first layer. The overall bilayer tilt of the carboxylic acidterminated thiols thus amounts to approximately $60^{\circ}$ (a mean value with a considerable spreading for the longer thiols, see Fig. 2A). Remarkably, this is an angle that alkanethiols like to adopt according to the carbon chain-interlocking model (steps of $\sim 15^{\circ}$ ). ${ }^{21}$ Both thiols with an odd number of carbon atoms in their alkyl chain ( $\mathrm{HSC}_{7} \mathrm{COOH}, \mathrm{HSC}_{11} \mathrm{COOH}$ and $\mathrm{HSC}_{15} \mathrm{COOH}$ ), where the $\mathrm{COOH}-$ group is in plane, and thiols with an even numbers of carbon atoms in their chain $\left(\mathrm{HSC}_{10} \mathrm{COOH}\right.$ and $\left.\mathrm{HSC}_{14} \mathrm{COOH}\right)$, where the $\mathrm{COOH}$ group is out of plane, fit to this model. However, the lateral friction clearly differs characteristically between the patches of the 'odd' and 'even' thiols. The friction on patches of the 'even' thiols is slightly higher than that on the odd ones (Fig. 2B), while for the former thiols the measured variation in friction is $4-6$ times higher than for the latter. These observations indicate that bilayers of 'even' $\mathrm{HSC}_{n} \mathrm{COOH}$-molecules are less stable than bilayers of their 'odd' analogues, which can be rationalized by a larger sterical hindering between neighboring molecules upon the formation of $\mathrm{COOH}-$ HOOC cyclic dimers in the case of the 'even' $\mathrm{HSC}_{n} \mathrm{COOH}$-type thiols (Fig. 3A and B). 

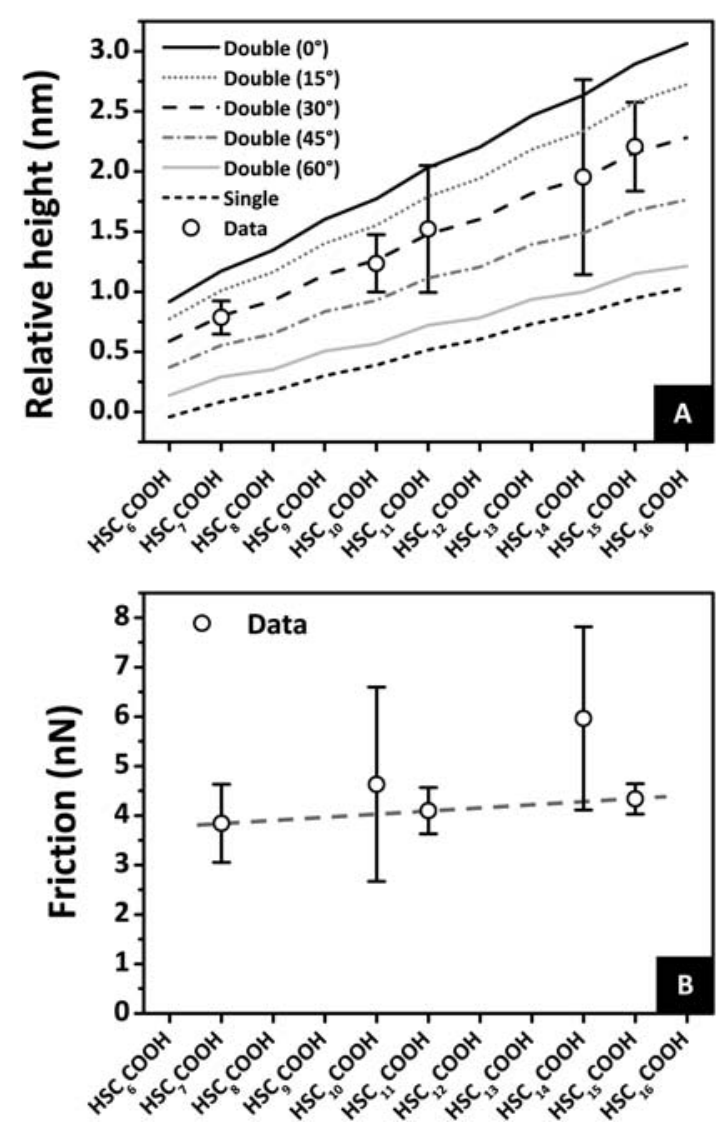

Fig. 2 (A) Relative height of nanografted patches of $\mathrm{HSC}_{n} \mathrm{COOH}$ $\left(n=7,10,11,14\right.$ and 15) in an $\mathrm{HSC}_{8}$ matrix. The different lines indicate the expected heights according to a single layer or a double layer model with different interlayer angles of $0^{\circ}, 15^{\circ}, 30^{\circ}, 45^{\circ}$ and $60^{\circ}$. (B) Corresponding lateral friction values of the nanografts. The trend line indicates the increase in friction observed for 'odd' $\mathrm{HSC}_{n} \mathrm{COOH}$ molecules.

All of the $\mathrm{HSC}_{n} \mathrm{COOH}$ nanografted bilayer patches exhibit an approximately 3 times higher friction than that of the monolayer patch of $\mathrm{HSC}_{11} \mathrm{OH}(1.36 \pm 0.47 \mathrm{nN}$, Fig. $2 \mathrm{~B}) \cdot{ }^{13}$ It can be expected that the bottom layer SAM bound to the gold surface is closely packed, as has been described before, ${ }^{6}$ and consequently the large increase in friction can be mainly attributed to the presence of the second layer of $\mathrm{HSC}_{n} \mathrm{COOH}$-molecules. This layer is expected to be more dynamic and less well stabilized by van der Waals interactions than the first one, leading to an imperfect packing. Further evidence for this greater instability is supplied by the slight increase in friction observed upon lengthening the alkyl chain of the $\mathrm{HSC}_{n} \mathrm{COOH}$ molecules (Fig. 2B, dotted trendline), which in conventional SAMs of thiols would lead to a better monolayer packing and lower friction values. ${ }^{1,22}$

To verify that the bilayer formation relies on $\mathrm{COOH} \cdots \mathrm{HOOC}$ and not on $\mathrm{COOH} \cdots \mathrm{HS}$ hydrogen bonding, nanografted patches were created of $\mathrm{HSC}_{10} \mathrm{COOH}$ and 9 equivalents of 1-hexadecanoic acid $\left(\mathrm{C}_{15} \mathrm{COOH}\right)$ or 1-octadecanoic acid $\left(\mathrm{C}_{17} \mathrm{COOH}\right)$ in 2-butanol. The alkanoic acids cannot bind to gold, but they are still able to form strong hydrogen bonds with a SAM of $\mathrm{HSC}_{10} \mathrm{COOH}$. The topographies of the resulting nanografted patches were slightly higher than those of the patches of the pure $\mathrm{HSC}_{10} \mathrm{COOH}$ thiols, and exactly matched a heterodimer bilayer of $\mathrm{HSC}_{10} \mathrm{COOH} \cdots \mathrm{HOOCC}_{15}$ and
$\mathrm{HSC}_{10} \mathrm{COOH} \cdots \mathrm{HOOCC}_{17}$, respectively, assuming the same angles as those of the homodimer bilayer (Fig. S1 $\dagger$ ).

The relative height of $2.28 \pm 0.40 \mathrm{~nm}$ found for the nanografted patch of $\mathrm{HSC}_{11} \mathrm{NH}_{2}$ (Fig. 1D) also indicates bilayer formation. In this case, fitting to our model suggests that the top layer of molecules is oriented at an angle of only $\sim 2^{\circ}$ with respect to the surface normal, and $-28^{\circ}$ with respect to the first layer (Fig. 3C). This different behavior in comparison to the $\mathrm{HSC}_{n} \mathrm{COOH}$-molecules can be explained by a difference in hydrogen bonding strength and geometry. It is expected that the $\mathrm{HSC}_{11} \mathrm{NH}_{2}$ molecules form single hydrogen bonds between their amino groups, which provides them with more degrees of freedom than the $\mathrm{HSC}_{n} \mathrm{COOH}$-molecules and which results in an upright alignment of the molecules. The weaker hydrogen bonds between the amino-functionalized thiols will probably lead to a more disordered top layer, which is directly reflected in the higher friction of the nanografted patch (Fig. 1E).

The observation that $\mathrm{HSC}_{11} \mathrm{OH}$ does not form similarly stable bilayers as the $\mathrm{NH}_{2-}$ and $\mathrm{COOH}$-terminated thiols are remarkable, also because $\mathrm{O}-\mathrm{H} \cdots \mathrm{O}$ hydrogen bonds are generally stronger than $\mathrm{N}-\mathrm{H} \cdots \mathrm{N}$ ones. This apparent inability to form interlayer hydrogen bonds might be attributed to an unfavorable competition with the formation of strong hydrogen bonds between the $\mathrm{HSC}_{11} \mathrm{OH}$ molecules within the SAM, or an unfavorable competition with the solvent for hydrogen bonding.

To investigate the mechanical stability of the second layer, the force applied by the probing AFM tip was increased. First, the response of a bilayered nanografted patch of $\mathrm{HSC}_{10} \mathrm{COOH}$ was probed over time under an ethanol supernatant at a load of $12 \mathrm{nN}$ (Fig. 4). Within 9 scans ( $t=12-55 \mathrm{~min})$ the top layer is lost and the resulting nanografted patch is just $0.35 \pm 0.22 \mathrm{~nm}$ high, which is in good agreement with the predicted height of a monolayer of $\mathrm{HSC}_{10} \mathrm{COOH}(0.39 \mathrm{~nm})$ (Fig. 4A, $t=52 \mathrm{~min}$, and Fig. 4B). After lowering the load to $4 \mathrm{nN}$, the bilayer structure reforms rapidly (Fig. 4B, $t=55-72 \mathrm{~min}$ ), mainly indicated by the restoration of the height to a value observed before removal of the second layer. The disappearance of the second layer is accompanied with a clear decrease in lateral friction, again confirming that the SAM is more tightly and uniformly packed than the bilayer (Fig. 4C). Analysis of the surface roughness of the bilayer compared to that of the monolayer $\left(R_{\mathrm{q}}\right.$-values of $0.21 \pm 0.03 \mathrm{~nm} v$ v. $\left.0.17 \pm 0.04 \mathrm{~nm}\right)$ indicates that its influence on the friction will be minimal.

When the same stability test of an $\mathrm{HSC}_{10} \mathrm{COOH}$ nanografted patch was carried out in 2-butanol instead of ethanol, the second layer remained completely intact and could even resist forces up to $\sim 30$ $\mathrm{nN}$. This observation reveals the direct influence of the supernatant solvent on the bilayer stability, which is probably for an important part based on its role as competitor for the $\mathrm{COOH}^{\cdots} \mathrm{HOOC}$ hydrogen bonding formation between the thiols. The hydrogen bonding strength of the secondary alcohol 2-butanol is weaker than that of the primary alcohol ethanol. For that reason the interlayer hydrogen bonds are significantly more stable in the former solvent than in the latter, resulting in a bilayer that remains inert at high load forces. In addition, ethanol is more hygroscopic than 2-butanol, which will contribute to a further destabilization of the bilayer in the former solvent.

In a similar experiment, the stability of an $\mathrm{HSC}_{11} \mathrm{NH}_{2}$ nanografted patch in 2-butanol was investigated (Fig. S2†). Upon increasing the force load to $12 \mathrm{nN}$, a decrease in height and friction was observed and within 5 scans the second layer had completely disappeared. 


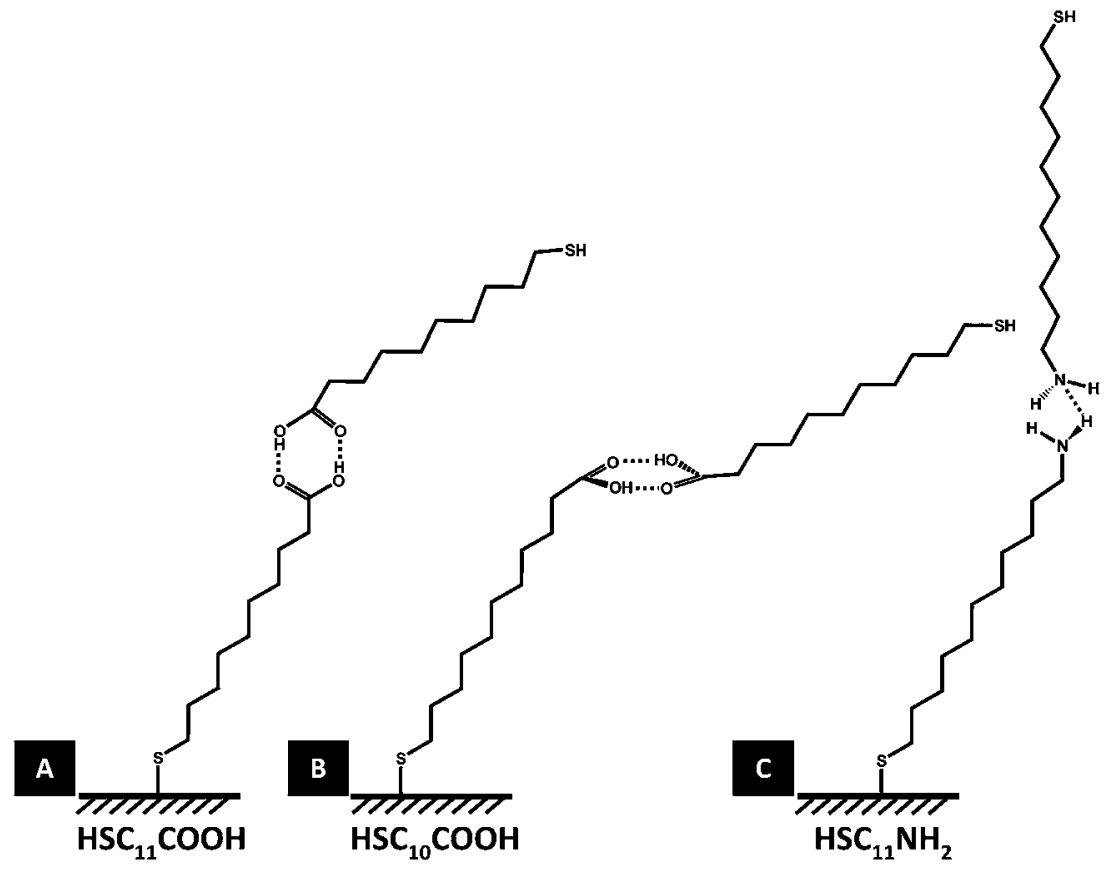

Fig. 3 Model of the formation of double layers stabilized by hydrogen bonds between the amine and carboxylic acid groups. (A) A double layer between two 'odd' $\mathrm{HSC}_{n} \mathrm{COOH}$-thiols (in this case $\mathrm{HSC}_{11} \mathrm{COOH}$ ), the $\mathrm{COOH}$-group is out of plane. (B) A double layer between two 'even' $\mathrm{HSC}_{n} \mathrm{COOH}$-thiols (in this case $\mathrm{HSC}_{10} \mathrm{COOH}$ ), the $\mathrm{COOH}$-group is in plane, and $90^{\circ}$ rotated. (C) A double layer of $\mathrm{HSC}_{11} \mathrm{NH}_{2}$ formed via a single hydrogen bond.
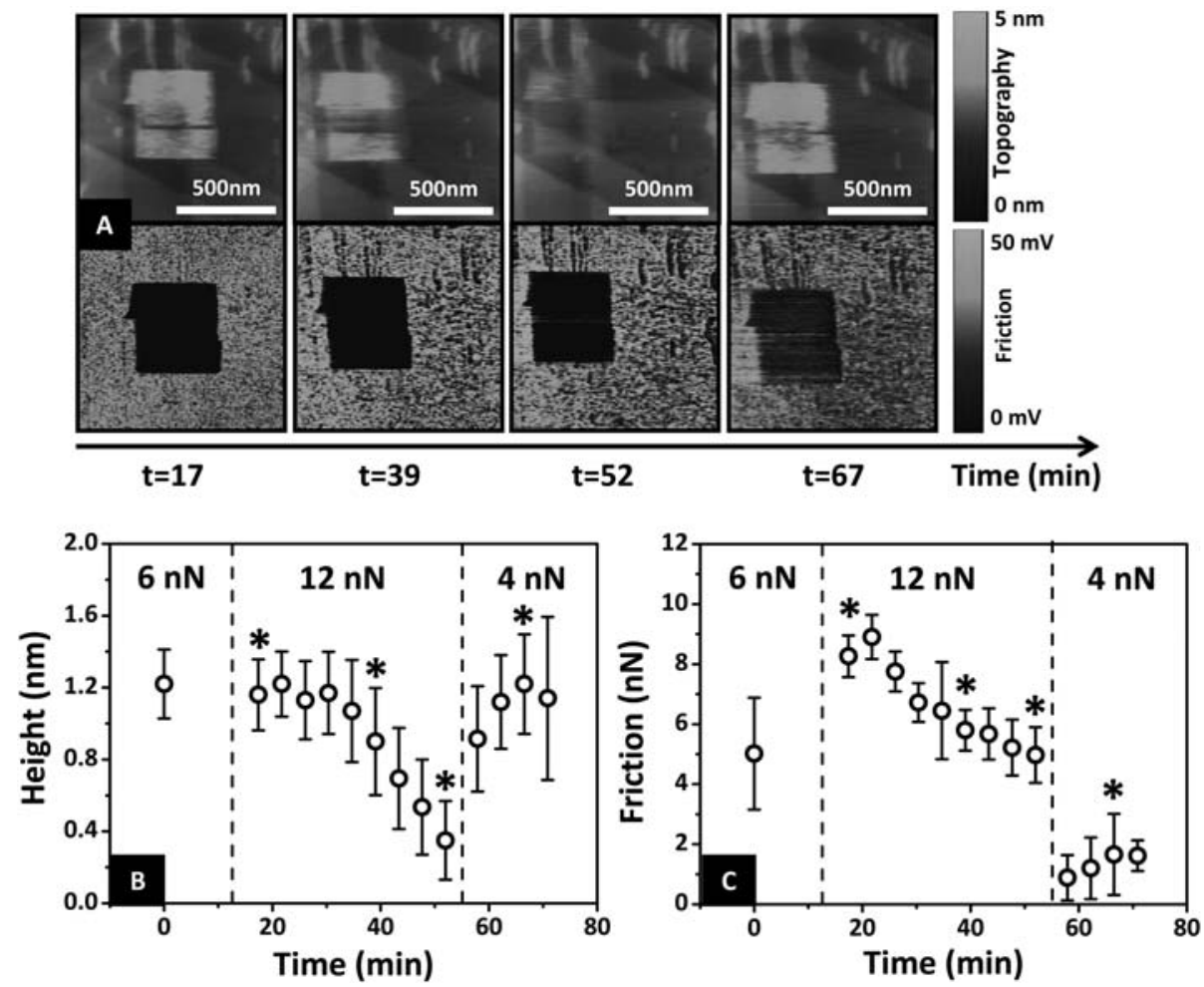

Fig. 4 Mechanical stability test of a nanografted patch of $\mathrm{HSC}_{10} \mathrm{COOH}$ in a $\mathrm{HSC}_{8}$ matrix performed by varying the load performed with the AFM tip. The tip scan speed is kept constant at $6 \mu \mathrm{m} \mathrm{s}^{-1}$. (A) Series of topography and friction images following an $\mathrm{HSC}_{10} \mathrm{COOH}$ nanografted patch in an ethanol subphase in time. (B) Height variation of the nanografted patch in time. The nanografted patch was created and imaged at $t=0 \mathrm{~min}$ at a $6 \mathrm{nN}$ load, at $t=12 \mathrm{~min}$ the load was increased to $12 \mathrm{nN}$, and at $t=55 \mathrm{~min}$ decreased to $4 \mathrm{nN}$. (C) Corresponding friction values of the nanografted patch in time. The images in (A) were measured at the time points marked with an *. Note that the values of the friction also depend on the applied load force. 
Upon subsequent lowering of the load, in some cases the second layer did not return $(\sim 30 \%)$. Both observations confirm our earlier hypothesis that the hydrogen bonding between $\mathrm{NH}_{2}$-functionalized thiols in a bilayer structure is weaker than that between $\mathrm{COOH}$ functionalized thiols, which reformed in all cases. Further proof for this behavior comes from an experiment in which a nanograft of $\mathrm{HSC}_{11} \mathrm{NH}_{2}$ was constructed in ethanol. In the AFM image (Fig. S3†), a relative height of the patch of $0.46 \pm 0.15 \mathrm{~nm}$ was measured, which is in good agreement with the formation of only a monolayer structure of this thiol.

In summary, alkanethiols with $-\mathrm{NH}_{2}$ and $-\mathrm{COOH}$ endgroups form stable bilayer structures when they are grafted in a SAM matrix from a 2-butanolic supernatant solution. The two layers are kept together by hydrogen bonds, in the form of cyclic dimer bonds between $\mathrm{COOH}$-terminated thiols or as single bonds between $\mathrm{NH}_{2}-$ terminated thiols. The high friction observed for double layered nanografts is attributed to disorder in the top layer. Furthermore, in the case of 'odd' and 'even' $\mathrm{HSC}_{n} \mathrm{COOH}$-molecules the friction slightly fluctuates due to differences in intermolecular interactions. Finally, the concentration of the molecules and the type of supernatant solution, e.g., ethanol, 2-butanol, or water, strongly influences the stability of the bilayer structures by competing with the hydrogen bond formation between the $\mathrm{COOH}$ - and $\mathrm{NH}_{2}$-groups of the alkanethiols.

\section{Acknowledgements}

This study is supported by NanoNed, the Dutch nanotechnology programme of the Ministry of Economic Affairs. A.C. is supported by a VENI (916.66.028) and MEERVOUD (836.09.002) grant of the Netherlands Organization for Scientific Research (NWO). J.A.A.W.E. thanks the Council for the Chemical Sciences of the Netherlands Organization for Scientific Research (CW-NWO) for a VIDI grant (700.58.423). C.G.F. is supported by a BIO-LIGHTTOUCH grant (FP6-2004-NEST-C-1-028781) and Immunanomap grant (MRTN-CT-2006-035946) of the European Union. Arend M. van Buul is acknowledged for kindly providing us with 11-amino-1undecanethiol.

\section{References}

1 J. C. Love, L. A. Estroff, J. K. Kriebel, R. G. Nuzzo and G. M. Whitesides, Chem. Rev., 2005, 105, 1103.

2 G. E. Poirier and E. D. Pylant, Science, 1996, 272, 1145.

3 G. E. Poirier and M. J. Tarlov, Langmuir, 1994, 10, 2853.

4 S. Xu and G. Y. Liu, Langmuir, 1997, 13, 127.

5 J. E. Baio, T. Weidner, J. Brison, D. J. Graham, L. J. Gamble and D. G. Castner, J. Electron Spectrosc. Relat. Phenom., 2009, 172, 2.

6 S. M. Mendoza, I. Arfaoui, S. Zanarini, F. Paolucci and P. Rudolf, Langmuir, 2007, 23, 582.

7 H. Wang, S. F. Chen, L. Y. Li and S. Y. Jiang, Langmuir, 2005, 21, 2633.

8 R. Arnold, W. Azzam, A. Terfort and C. Woll, Langmuir, 2002, 18, 3980.

9 O. Dannenberger, K. Weiss, H. J. Himmel, B. Jager, M. Buck and C. Woll, Thin Solid Films, 1997, 307, 183.

10 R. G. Nuzzo, L. H. Dubois and D. L. Allara, J. Am. Chem. Soc., 1990, 112, 558.

11 M. Bergkvist, N. Niamsiri, A. D. Strickland and C. A. Batt, Surf. Sci., 2008, 602, 2121.

12 M. Liu, N. A. Amro and G. Y. Liu, Annu. Rev. Phys. Chem., 2008, 59, 367.

13 J. te Riet, T. Smit, J. W. Gerritsen, A. Cambi, J. A. A. W. Elemans, C. G. Figdor and S. Speller, Langmuir, 2010, 26, 6357.

14 S. Xu, P. E. Laibinis and G. Y. Liu, J. Am. Chem. Soc., 1998, 120, 9356.

15 S. Cruchon-Dupeyrat, S. Porthun and G. Y. Liu, Appl. Surf. Sci, 2001, 175-176, 636.

16 H. I. Kim and J. E. Houston, J. Am. Chem. Soc., 2000, 122, 12045.

17 H. I. Kim, T. Koini, T. R. Lee and S. S. Perry, Langmuir, 1997, 13, 7192.

18 Y. H. Liu, T. Wu and D. F. Evans, Langmuir, 1994, 10, 2241.

19 L. Shuang, P. Cao, R. Colorado, X. P. Yan, I. Wenzl, O. E. Shmakova, M. Graupe, T. R. Lee and S. S. Perry, Langmuir, 2005, 21, 933.

20 X. D. Xiao, J. Hu, D. H. Charych and M. Salmeron, Langmuir, 1996, 12, 235.

21 E. Barrena, C. Ocal and M. Salmeron, J. Chem. Phys., 2000, 113, 2413.

22 A. Lio, D. H. Charych and M. Salmeron, J. Phys. Chem. B, 1997, 101, 3800 . 